\title{
Occasion-sensitive semantics for objective predicates
}

\author{
Tamara Dobler ${ }^{1}$ \\ Published online: 14 March 2019 \\ (C) The Author(s) 2019
}

\begin{abstract}
In this paper I propose a partition semantics (Groenendijk and Stokhof in Studies on the semantics of questions and the pragmatics of answers, Ph.D. thesis, University of Amsterdam, 1984) for sentences containing objective predicates that takes into account the phenomenon of occasion-sensitivity associated with so-called Travis cases (Travis in Occasion-sensitivity: Selected essays, Oxford University Press, Oxford, 2008). The key idea is that the set of worlds in which a sentence is true has a more complex structure as a result of different ways in which it is made true. Different ways may have different capacities to support the attainment of a contextually salient domain goal. I suggest that goal-conduciveness decides whether some utterance of a sentence is accepted as true on a particular occasion at a given world. The utterance will not be accepted as true at a world which belongs to a truth-maker which is less conducive to a contextually salient goal than other truth-makers. Finally, the proposed occasionsensitive semantics is applied to some cases of disagreement and cancellability.
\end{abstract}

Keywords Occasion-sensitivity · Travis cases · Partition semantics · Truth-makers · Domain goals · Disagreement

\section{Introduction}

\subsection{Objective and discretionary predicates}

This paper presents a novel approach aimed at explaining the occasion-sensitivity of objective predicates. By objective predicate, I refer to a broad range of predicates such

This research is part of the project RContext (656273) Horizon 2020 Marie Sklodowska-Curie Actions funded by European Commission. I would like to thank Maria Aloni, Alun Davies, Alex Davies, Floris Roelofsen, Martin Stokhof, Robert van Rooij, two anonymous reviewers and the editors of this journal for their comments on earlier drafts of this paper.

\footnotetext{
$\bowtie$ Tamara Dobler

T.Dobler@uva.nl

1 Institute for Logic, Language and Computation, Universiteit van Amsterdam, P.O. Box 94242, 1090 GE Amsterdam, The Netherlands
} 
as green, desk, doctor, or under the bed, which can be contrasted with subjective or discretionary predicates (predicates of personal taste) such as tasty or fun (cf. Lasersohn 2005; Kölbel 2004; Coppock 2018). To properly appreciate the difference between these types of predicates consider the following pair of dialogues, the first involving the objective predicate doctor and the second involving the discretionary predicate tasty:

1. (a) A: John is a doctor.

(b) B: No, John is not a doctor.

2. (a) A: The chilli is tasty.

(b) B: No, the chilli is not tasty.

Intuitively, in the dialogue (1) either A or B (but not both) is mistaken, both can't be saying something true: there is a fact of the matter as to whether John is a doctor. By contrast, in the dialogue (2), both A and B could be saying something true; none is at fault whilst they seem to be disagreeing. ${ }^{1}$ Different accounts have been proposed to account for this intuitive contrast; what they seem to agree on is that the interpretation (evaluation) of a sentence containing a discretionary predicate is relative to certain subjective parameters (judge, centred world, outlook, perspective, etc.), which are taken to determine either its intension (contextualism) or its extension (relativism). By contrast, a simple factual statement containing an objective predicate is normally considered true at a world simpliciter without its intension or extension being dependent on further factors. ${ }^{2}$

\subsection{Travis cases}

The intuitive contrast between objective and discretionary predicates seems to break down in light of occasion-sensitivity. So-called Travis cases (Travis 1978, 2000, 2008, 2009) show that truth conditions (and/or evaluations) of factual statements could vary depending on certain factors even when the point of evaluation remains the same. In the following dialogue, for instance, we are asked to imagine that the leaves in question are naturally red but painted green. The artist needs green leaves for an artistic decoration, the botanist for an experiment. ${ }^{3}$

\footnotetext{
${ }^{1}$ For discussion of faultless disagreement see Kölbel (2004), MacFarlane (2014), Stojanovic (2007), Huvenes (2014) and Coppock (2018).

2 Unless, of course, a sentence thereby used contains indexical elements, in which case a context will need to supply contextual values for indexicals. Kaplan's informal definition of indexicals makes the key reference to a semantic rule associated with their meaning which determines their content in context: "What is common to the words or usages in which I am interested is that the referent is dependent on the context of use and that the meaning of the word provides a rule which determines the referent in terms of certain aspects of the context." (Kaplan 1989: 490) Since there is no comparable rule that governs the interpretation of objective predicates on particular occasions of use, I take it that they don't behave as indexicals. This observation also applies to accounts that postulate indexical elements in the noun phrase (see Vicente 2012; Giberman 2016). For discussion see Rothschild and Segal (2009), Kennedy and McNally (2010), Recanati (2004) and Travis (2008).

${ }^{3}$ In the original exposition of this case the following scenario is described:
} 
3. (a) The artist: The leaves are green.

(b) The botanist: No, the leaves are not green.

Presumably, green like doctor is an objective predicate: i.e. it is a matter of fact (rather than opinion or taste) as to whether something is green or not. However, in the dialogue (3) there is an intuitive sense in which neither the artist nor the botanist is at fault: relative to their occasions (goals) both could be saying something true. Still, by the initial assumption, insofar as there is a fact of the matter as to whether the leaves are green one of them ought to be at fault.

\subsection{Reinstating the contrast?}

Can we preserve the intuitive contrast between discretionary and objective predicates if objective predicates are occasion-sensitive in this way? A key aim in this paper will be to try to explain the phenomenon of occasion-sensitivity without compromising the objectivity of factual statements and the notion of objective disagreement. It will be argued that the sense of faultlessness in the dialogue (3) arises due to the fact that speakers modify their acceptance of when the sentence is true in an attempt to fulfil certain practical goals. Depending on what this goal is, the sentence may or may not be accepted as true for the purposes of that goal. Although the interpretation of both objective and discretionary predicates is, on this account, relative to some (contextual) factors, the interpretation of tasty is relative to subjective or personal factors (i.e. something to do with $\mathrm{A}$ and $\mathrm{B}$ as individuals) and the interpretation of green or doctor is relative to objective factors, i.e. something to do with what A (artist) and B (botanist) do, with their plans, goals and actions.

\subsection{The current approach and the roadmap}

Let's briefly outline the main points that are crucial to the approach defended in this paper. In an attempt to accommodate the intuitions elicited by Travis cases, I defend a view that, despite having an important role in the interpretation of an utterance, our knowledge of standing meaning must be supplemented by other extralinguistic factors which, besides meaning, play a role in our intuitive evaluations of utterances. ${ }^{4}$ Knowing the meaning of a sentence leaves room for a great deal of variation among the truth-makers for a sentence: for instance, the sentence The leaves are green is true when the leaves are only painted green, but also when they are only naturally

Footnote 3 continued

A story. Pia's Japanese maple is full of russet leaves. Believing that green is the colour of leaves, she paints them. Returning, she reports, 'That's better. The leaves are green now.' She speaks truth. A botanist friend then phones, seeking green leaves for a study of green-leaf chemistry. 'The leaves (on my tree) are green,' Pia says. 'You can have those.' But now Pia speaks falsehood (Travis 2008: 111).

4 This position has been defended for more than four decades by Charles Travis and others. See Travis (1978, 2008), Moravcsik (1994), Recanati (2004), Carston (2002), Austin (1975), Searle (1980), Chomsky (2000) and Pietroski (2005). 
green, or both. ${ }^{5}$ Whereas all these different sets of worlds make the sentence true they nonetheless do so in different ways. The key point is that these differences may matter for a successful fulfilment of a given goal, and thus some alternatives may be preferred to some others. In order to capture the idea that an atomic sentence may have a set of alternative truth-makers, in Sect. 2 I propose an approach inspired by a partition semantics for questions (Groenendijk and Stokhof 1984). On this approach, the conventional, standing or literal meaning of a sentence determines a very broad, undifferentiated range of worlds at which the sentence is true (henceforth, the truth set for $p$ ). However, this set can be further partitioned according to alternative ways in which $p$ is true, where each partition cell corresponds to a different truth-maker for ${ }^{6}{ }^{6}$

Among extralinguistic factors, contextually salient domain goals (Roberts 1996, 2012) are crucial in restricting the set of truth-makers (a partition of the truth set for $p$ ) to those most likely to support a particular course of action. In Sect. 3 I introduce the notion of goal-conduciveness, which is a feature that agents assign to partition cells (truth-makers) on the basis of whether they judge them to be conducive to their goals. If a particular truth-maker for a sentence is less conducive to a given domain goal then it won't be recognised by rational, well-informed agents as a truth-maker of that sentence on the occasion where the goal is salient. As a consequence, the utterance of the sentence is considered false on that occasion. Thus, to understand correctly what someone has said presupposes being aware of a salient domain goal, because goals determine which particular ways that the world could be, out of many compatible with the standing meaning of a sentence, are in the agent's purview on that occasion. In this way pragmatics decides how a sentence ought to be interpreted if we are to carry out our practical projects.

The current account belongs to the family of approaches that explain occasionsensitivity by appealing to processes of free pragmatic enrichment (see Recanati 2004, 2010; Pagin 2005; Pagin and Pelletier 2007). However, the account also goes beyond standard enrichment approaches in that it replaces a generic modulation function with the domain goal function (and goal-conduciveness valuations) thus forging a more concrete connection between the possibilities denoted by a sentence (represented by partition cells) and different practical projects.

After describing a formal model in Sect. 4, in Sect. 5 I turn to two applications, namely, to intuitions about genuine and faultless disagreement (5.1) and to cancellability (5.2).

\footnotetext{
5 I follow Yablo $(2014,2017)$ in defining a truth-maker for a sentence as a fact which makes the sentence true, formally represented as a set of possible worlds. However, unlike Yablo, I also allow that an atomic sentence $p$ can have more than one truth-maker: $p$ can be made true in more than one way. Although the union of truth-makers of $p$ forms a cover over the the set of worlds where $p$ is true, the two sets (the set of $p$ 's truth-makers and $p$ 's truth set) are not identical.

6 The point is similar to the one Yablo makes vis-a-vis disjunctive sentences: "When Frost writes, The world will end in fire or in ice, the truth-conditional meaning of his statement is an undifferentiated set of scenarios. Its 'enhanced' meaning is the same set, subdivided into fiery-end worlds and icy-end worlds." (Yablo 2014: 2)
} 


\section{Partition semantics for simple declaratives}

What does one know when one knows the standing meaning of an objective predicate and a declarative sentence containing it? What does knowing the meaning of a declarative sentrence enable one to do? A classical answer is that to know the meaning of a sentence is to be able to specify its truth conditions, i.e. when it would be true. Travis cases bring this idea into question by showing that knowing meaning and the world of evaluation doesn't guarantee an agreement between two agents as to whether the sentence is true. Our challenge then is to adequately explain the idea that although meaning does place some restrictions on which worlds are candidates for making an utterance of a sentence is true, it does not go so far as to determine, for any given world, whether we would accept it as an instance of a truth-maker for the utterance.

\subsection{Ways of being $F$}

The idea that there are different ways in which a sentence is true reflects the fact that we often use an objective predicate in several different senses. ${ }^{7}$ The predicate green, for instance, can be (non-figuratively) used in the sense of naturally green, painted green, lit green, mouldy green and so on. Insofar as a predicate can be used in different senses, I assume that its wide denotation also has a more complex structure which I will refer to by talking of different ways of being $F{ }^{8}$ Travis cases are specially designed to distinguish ways of being $\mathrm{F}$ by highlighting that a certain way may be more prominent to the speakers on a given occasion, compared to other such ways. ${ }^{9}$

I think Travis cases succeed at showing us that there can be different ways of being $\mathrm{F}$, and I also think that in the context of those cases, what constitutes a way of being $\mathrm{F}$ is comprehensible. That said, questions loom about how exactly ways get distinguished from one another, whether some ways have precedence over others, whether there are limits on the number of ways, and so on. For the purposes of what I want to achieve here, I want to briefly present my take on two apposite issues.

First, when speaking of ways, I take a metaphysically light view that no particular way of being $\mathrm{F}$ has precedence over any other in terms of its being a more 'strictly speaking' or 'proper' way of being F. For instance, questions may arise as to whether an object that is lit green or painted green is actually green at all. The kind of metaphysical debate that might ensue from such questions, however, is not one I wish to engage with here. For present purposes, the fact that there are conceivable occasions where individuals would (felicitously) speak of painted green or lit green leaves as being green is enough to suggest that these can be accepted as ways of being green. My

\footnotetext{
7 This section addresses a number of issues raised by an anonymous reviewer who I wish to thank for pressing me to clarify my position regarding the notion of ways.

${ }^{8}$ I use $a, b, c \ldots$ to denote individuals, F, G ... to denote properties. For atomic sentences, I use Fa, Gb and sometimes also sentential variables $p, q, r$.

9 Note that ways of being $\mathrm{F}$ are not like types of F. We can sort green objects into different types of greeni.e. emerald green, lime green, and so on. Equally, we can sort those objects as being green in different ways-i.e. lit green, painted green, and so on. But there's a difference of kind between ways and types. Types of green are not ways of being green, and lit green is not a type of colour green.
} 
motivation for introducing the notion of ways of being $\mathrm{F}$ is thus primarily linguistic, not metaphysical. ${ }^{10}$

Second, it might be suggested that once we acknowledge ways, it's hard to set any kind of limit on the ways in which an object may count as being F. A forteriori, anything could count as being F on some way of understanding F. To my mind, though, this worry is unfounded for the following reasons.

Reason one It's not the case that anything can count as being F. For instance, whatever the occasion, the glass of water in front of me, as it is right now (present light conditions, position, etc.), would no more pass for being green than it would pass for being a piano or a cow. And this isn't because there's some hard-to-recognise way in which the glass is green that is being missed here. Indeed, there's no reason to think that ways of being F are ever hard to recognise. The botanist doesn't count painted green leaves as green leaves, but that doesn't mean she doesn't recognise that someone might count being painted green as a way for the leaves to be green. So, assuming that ways of being $\mathrm{F}$ are relatively easy to recognise, especially when one is looking for them, we can assume that the glass on my desk is not green in any way.

Reason two Ways of being F are not easily multiplied. In particular, one can't split ways of being F merely by pointing to the contingencies that led to an object becoming F. Consider two imaginary colour machines, machine A and machine B, whose settings differ only in the following respect: A turns red and green leaves blue, but purple and yellow leaves green; B turns red, green and yellow leaves blue, but only purple leaves green. ${ }^{11}$ Would being acted upon by one machine give us one way of being green, and being acted on by the other another? I would argue not. There's arguably no Travis case to be made for someone counting leaves acted on by machine A as green whilst simultaneously counting leaves acted on by machine B as not green. The relevant difference in the internal settings of the machines appears to be something a good Travis case would abstract away from and not a difference upon which we can split ways.

Both of these points support the idea that there are limits on ways of being $\mathrm{F}$ even when they are not grounded in metaphysics. Travis cases are constructed at a sufficient level of abstraction to ensure they don't overgenerate ways. And ways are easy enough to recognise that we don't need to worry about there being lots of not-yet-revealed ways in which all the objects around us are F. There are of course other questions to be asked about ways, but for present purposes, I hope to have addressed some of the pressing worries that arise with regards to this notion.

\subsection{Truth-conditions and alternatives}

Let us call the set of worlds where an atomic sentence $p$ is true, the truth set for $p$, or $|p|$ for short. Anyone who knows the meaning of $p$ knows the minimal proposition expressed by $p$, and, on the basis of this knowledge, can expect the actual world to be

\footnotetext{
10 I don't want to speculate about what greenness in a strictly speaking, metaphysically justifiable sense might be, but leave it open that there is such a thing.

11 I owe this example to the anonymous reviewer.
} 
somewhere in $|p|$ when $p$ is asserted. ${ }^{12}$ To capture the notion that one's knowledge of a minimal proposition is not sufficient to determine in which way $p$ is true, we can use partition semantics to represent alternatives that are not resolved in virtue of grasping the minimal proposition. Each cell of the partition of $|p|$ represents an alternative way for $p$ to be true (for the sentence $\mathrm{Fa}$, the partition of $|\mathrm{Fa}|$ is induced by different ways of being F). Although in question semantics partitions are typically used to represent possible answers to a question (see Groenendijk and Stokhof 1984), the idea of meaning as a set of alternatives is preserved in the current account in the form of alternative truth-makers for an atom. Whilst determining when $p$ is true, meaning nevertheless leaves it undetermined in which way $p$ is made true. Accordingly, we can think of $p$ 's alternatives as a function from a set of worlds where $p$ is true to a partition $\mathrm{P}$ of $|p|$ such that each individual cell of $\mathrm{P}$ corresponds to an alternative way for $p$ to be true (i.e. a truth-maker for $p$ ).

Definition 1 (Meaning, truth-conditions) The meaning of an atomic sentence $p$ is a function from possible worlds to truth values, or equivalently, the set of worlds where $p$ is true, $|p|$.

Definition 2 (Alternatives, truth-makers) Alternatives for $p$ represent a function from $|p|$ to a partition $\mathrm{P}$ of $|p|$ such that each cell of $\mathrm{P}$ corresponds to an alternative way in which $p$ is made true. ${ }^{13}$

To illustrate let's assume there are only two basic ways of being F, where $a$ may be $\mathrm{F}$ in only one way or both ways at once. Then the partition of $|\mathrm{Fa}|$ will contain three cells such that their union forms a cover over $|\mathrm{Fa}|$. I depict $|\mathrm{Fa}|$ and the partition of $|\mathrm{Fa}|$ in Fig. 1a and b, respectively. The diagram in Fig. 1b thus represents the set of all alternative ways in which Fa is true (i.e. the set of truth-makers for Fa). Cell 11 consists of the worlds in which $a$ is F in both ways at once, cell 10 of the worlds in which $a$ is $\mathrm{F}$ in only one way, and cell 01 contains the worlds in which $a$ is $\mathrm{F}$ in the other way only. Since these exhaust the ways for $a$ to be F, the 00 cell consists of worlds in which $a$ is not $\mathrm{F}$. Note that, in some cases, the ways for $a$ to be F will be incompatible in which case cell 11 will not be part of a partition. ${ }^{14}$

\section{Goals and occasion-sensitivity}

In this section I look more closely at a pragmatic mechanism behind the exclusion of certain partition cells from the contextual proposition which explains why speakers find some of them unacceptable as truth-makers for an utterance as indicated in Travis cases. I propose to explain occasion-sensitivity as the sensitivity to domain goals

\footnotetext{
12 To some extent this is in line with the view known as semantic minimalism. For the defence of this view see Cappelen and Lepore (2005) and Borg (2004, 2012). As Emma Borg notes, "[the minimalist] holds that word meanings generalise over specific contextual understandings of them-that the meaning of 'green' leaves open the way in which an object might be green (on its surface, on the inside, etc.)" (Borg 2012: 46).

13 The partition of $|\mathrm{Fa}|$ is induced by ways of being F. See Sect. 4 for a formal treatment.

14 For instance, a door can be closed by being locked or by being unlocked. But these two ways for a door to be closed are incompatible and cannot be predicated of the same door at once.
} 


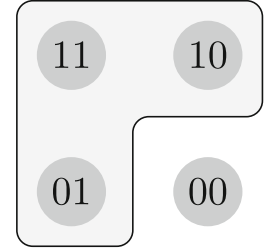

(a) $|\mathrm{Fa}|$

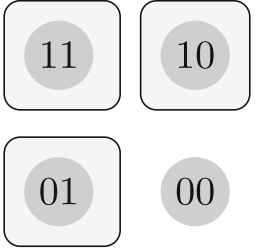

(b) Partition of $|\mathrm{Fa}|$

Fig. 1 The minimal proposition expressed by Fa and a tripartite partition

(Roberts 2012). The main idea is that, often, the sentence's truth-makers have different degrees of conduciveness to a given domain goal. Whether they are more or less goalconducive has an impact on the interpretation of an utterance.

\subsection{Domain and discourse goals}

A domain goal is a practical goal related to successfully performing a given activity such as fixing a ship, making an experiment, forming a committee, furnishing an office, hiding shoes, helping an impoverished student, or avoiding something unwanted, etc. Besides a discourse goal which is to stay on topic and settle a given question under discussion (QUD), interlocutors also strive to achieve a certain domain goal around which their conversation is structured. According to Roberts (2012),

[w] can characterize sincere, competent and cooperative interlocutors as holding two kinds of goals at any given point in a discourse, their discourse goals_-aiming to address particular questions in the QUD-and the rest, their domain goalsintuitively, those things they want to accomplish in the world (as opposed to their narrowly discourse goals). (Roberts 2012: 6)

For the present analysis, the distinction between discourse goals and practical domain goals is important. According to one recent account, discourse goals (i.e. resolving contextual QUDs) are seen as guiding the interpretation of occasion-sensitive declarative sentences:

[w] hat is said by a sentence in a context is the answer that it provides to a relevant question that is antecedently accepted as the topic of discussion. (Schoubye and Stokke 2015: 760)

Whereas (Schoubye and Stokke 2015) take the question-answer structure as a basis for their analysis of occasion-sensitivity, I instead suggest that there is a link between a domain goal that agents strive to achieve on an occasion and how they are inclined to interpret both what is asserted and what is asked. ${ }^{15}$ Whilst a discourse goal deter-

15 There is a similarity between Schoubye and Stokke's (2015) and the present account in that both use same formal models developed in question semantics to analyse occasion-sensitivity. Due to reasons of space, I can't go into an extensive comparison of the two accounts. One key difference is that in Schoubye and Stokke's account partitions represent possible answers to QUDs (as in Groenendijk and Stokhof 1984), 
mines which answer is relevant (as a resolution of a QUD) and on-topic, a domain goal determines which ways for things to be constituting this information are most prominent to the speakers in a context.

\subsection{Goal-conduciveness}

A domain goal provides a very tangible thing at stake that motivates interlocutors to favour those possibilities that are maximally conducive to that domain goal. For instance, we are practically motivated not to count a painted green leaf as green if we want to conduct an experiment. So domain goals create preferences (biases) for certain ways for $a$ to be $\mathrm{F}$ because not all of them are equally conducive to a given goal. The leaves being non-naturally green is a way for the leaves to be green, but this way of them being green is not conducive to the botanist's goal, and so it doesn't count as such for this purpose. That is, as a way for the leaves to be green, its prominence amongst other such ways is very low.

Since there are two types of goals in a conversation, there are also two types of conduciveness to a goal, namely, conduciveness to a domain goal and conduciveness to a discourse goal. However, to avoid confusion, I call the former goal-conduciveness and the latter relevance. Relevance as a property of conversational moves (utterances) is measured against a discourse goal and a topic of discussion (which can be expressed in the form of a question or not). ${ }^{16}$ Conversational moves are relevant just in case they (partially) answer a question (a move is an assertion) or they form part of a strategy to answer a question (a move is another question). ${ }^{17}$ If a question is a polar question, then, intuitively, both positive and negative answers are equally relevant and on-topic. However, not all relevant answers to a QUD must denote goal-conducive ways for things to be: the information that the leaves are not naturally green does resolve the botanist's query but this way for the leaves to be green to be is not conducive to her domain goal. The distinction is subtle but nonetheless crucial because, on the proposed analysis, goal-conduciveness (rather than relevance) determines what is said.

\subsection{Valuation maps}

For any domain goal there will be a valuation map (henceforth, c-map) that assigns each cell a goal conduciveness value 1 or 0 (henceforth, c-value). ${ }^{18}$ Ultimately, this value will depend on whether a particular way for $a$ to be $\mathrm{F}$ is conducive to the goal. ${ }^{19}$

\footnotetext{
Footnote 15 continued

whereas I use partitions to represent alternative truth-makers. Although contextual goals on both accounts determine what is said, on S\&S's view a contextual goal means a discourse goal (to resolve a QUD) and on my account it means a domain goal (to successfully perform a given practice or activity). I show how a new interpretation is generated via goal-conduciveness; in S\&S an intended interpretation is determined by a QUD (whilst the intended interpretation of the question under discussion is presupposed).

16 For discussion of the connection between topic-sensitivity and question-sensitivity see Hawke (2017).

17 See Roberts (2012) and Schoubye and Stokke (2015).

18 For the simplicity's sake, I assume only two values. However, it is clear that conduciveness to a goal is a matter of degree.

19 For a formal treatment see Sect. 4.2.5.
} 
A pragmatically competent agent will activate the relevant c-map once they have taken into account the salient goal.

Let $s$ be an information state modelled as a set of possible worlds that are compatible with the information available in the state. The information state of a semantically competent agent who believes that the sentence $p$ is true contains the truth set for $p$. For example, anyone who knows that the leaves are green knows that they are green in some way or other. Let us call the information state of an agent who would locate the actual world in any cell of the truth set for $p$, the initial information state. Besides the knowledge of meaning, the agent's information state also contains a certain amount of real world knowledge (e.g. about what a botanist is and why they might need green leaves for etc.) that is necessary for producing an accurate c-map. Of course, for some more specialised goals a more specialised knowledge base will be required which not everyone will share. ${ }^{20}$ In many cases, however, common-sense knowledge will suffice for producing a good c-map, and it will usually be presupposed in a discourse that the agent has activated the relevant map once the domain goal is made salient. ${ }^{21}$

The information state of a pragmatically competent agent is enhanced compared to the initial information state, in as much as the agent has taken into account the contextually salient goal and activates the relevant c-map. Which particular map is relevant on an occasion of utterance is thus determined by the salient domain goal on that occasion. Figure 2a illustrates the partition of the set of worlds where the leaves are green assuming two (compatible) ways for the leaves to be green; i.e. painted and naturally green. In cell 11 the leaves are both naturally and painted green, in cell 10 only painted green, and in cell 01 only naturally green. In Fig. 2b, I depict the c-map for the botanist's goal where cells 11 and 01 have maximal c-values for this goal. Figure 2c depicts the proposition expressed by The leaves are green in the botanist context, which only takes into account the cells with maximal c-values. Although there could be some variations among c-maps between agents (due to different knowledge bases), in many cases, pragmatically competent agents will have overlapping valuations as to which cells are conducive to a particular goal. As we shall see in Sect. 5.1, a prerequisite for genuine disagreement is that both agents have access to similar or overlapping valuations, otherwise they will not express the same proposition (i.e. they will communicate at cross-purposes).

\subsection{Communication}

On the current approach, not every utterance of a declarative sentence will be sufficiently informative in a certain context. Assuming the context of an utterance is

\footnotetext{
20 In those cases communication will be at cross-purposes (unless more information is explicitly provided). For discussion of the notion of disagreement by talking at cross-purposes, see Davies (2017).

21 Notice that pragmatic competence doesn't require that the agent knows how to achieve every single goal in all of its aspects (that's too strong, of course) only to be able to c-evaluate partitions of worlds in light of that aspect of the goal concerning which enough common-sense knowledge can be presupposed. For instance, assuming a certain level of general, common-sense knowledge about what a botanist does, an interlocutor (who herself is not a botanist) should be able to evaluate the worlds with naturally green leaves as more conducive to the goal than the worlds with non-naturally green ones, without however having to know other facts about how to successfully perform an experiment with the leaves. Thanks to an anonymous reviewer for an invitation to clarify this point.
} 


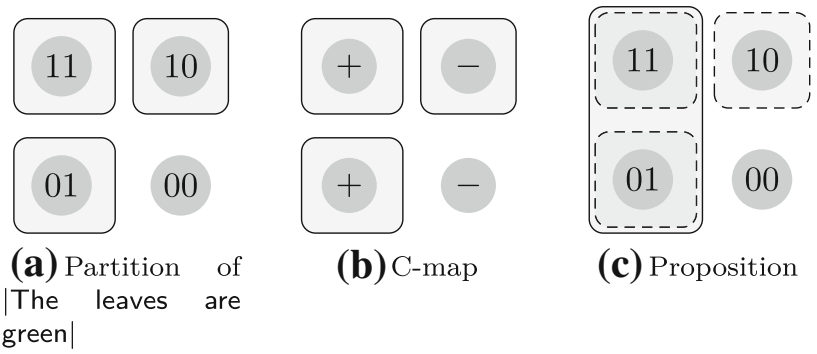

Fig. 2 Botanist context: partition, map and the proposition expressed

enriched with a domain goal, an assertion is considered sufficiently informative only if it has a bearing on that goal. ${ }^{22}$ To illustrate, consider the following polar question in (4). Let us assume that A, B, C, D and E are all semantically competent and know that there are different ways for the leaves to be green. Furthermore, they all know the contextually salient goal and have activated the relevant c-map.

\section{A: Are the leaves green $_{\gamma}$ ?}

(a) B: The leaves are green ${ }_{\gamma}$ or the leaves are not green . $_{\gamma}$

(b) $\mathrm{C}$ : The leaves are green (in some way or other).

(c) D: The leaves are green ${ }_{\gamma}$.

(d) E: The leaves are not green $_{\gamma}$.

A's question is uttered in a context where a certain goal $\gamma$ is operative and so it requires the c-map that is relevant to $\gamma$. The informativeness of an answer increases as more alternatives are excluded. (4a) is not informative since it asserts something that any rational agent should already know. (4b) excludes some possibilities, and to this extent it is not entirely trivial and uninformative. Yet it is not sufficiently (pragmatically) informative to facilitate a particular course of action or support A's goal because (4b) is compatible with any cell in the truth set. Those who seek information often do so with a particular end in sight. The answer in (4b) does not resolve a particular goalrelated issue; it does not contain the information based on which agent A can act. The only sufficiently informative answers that resolve the issue raised by the question in (4) are (4c) and (4d) because it is asserted (denied) that the actual world belongs to the truth-maker that is most conducive to $\gamma \cdot{ }^{23}$ An utterance of a negative sentence in (4d) can be seen as conveying the information that is indeed relevant vis-a-vis the issue raised in (4) and only indirectly conducive to the botanist goal although it does not have any goal-conducive sets of worlds as part of its denotation.

In the following section I define a formal model of occasion-sensitive semantics for a small fragment of English.

\footnotetext{
22 This is in line with some decision-theoretic approaches to questions where whether an answer resolves a question or not is determined by the salient decision problem. See Van Rooy (2003).

23 The notion of issues and their resolution conditions have been formalised in the framework of inquisitive semantics. See Groenendijk and Roelofson (2009), Ciardelli et al. (2013, 2015) and Ciardelli and Roelofsen (2015).
} 


\section{Occasion-sensitive semantics}

In order to determine when an utterance of a sentence $p$ on some occasion is considered true, I propose to evaluate the cells of the partition of the worlds where $p$ is true in terms of how conducive they are to the contextually salient domain goal $\gamma$, and to assign each cell a c-value. A set of maximally goal-conducive truth-makers of $p$ constitute the proposition expressed by the utterance of $p$ on an occasion where $\gamma$ is salient.

\subsection{Language}

A small language fragment for occasion sensitive semantics $\mathcal{L}_{o s}$.

\subsubsection{Basic expressions}

5. Individual constants: Sid, the leaves, the shoes.

6. Predicates: green, desk, under the bed.

7. Logical constants: $\neg, \wedge, \vee$.

\subsubsection{Formation rules}

8. if $\alpha$ is a predicate and $\beta$ is an individual constant, then $\alpha(\beta)$ is a sentence.

9. if $\phi$ is a sentence, then $\neg \phi$ is a sentence.

10. if $\phi$ is a sentence and $\psi$ is a sentence, then $\phi \vee \psi$ is a sentence.

11. if $\phi$ is a sentence and $\psi$ is a sentence, then $\phi \wedge \psi$ is a sentence.

\subsection{Occasion-sensitive semantics for $\mathcal{L}_{\text {os }}$}

A model $M$ for $\mathcal{L}_{o s}$ is a tuple $\langle D, W, I, \Sigma, \Gamma\rangle$ where

i. $D$ is a non-empty set of individuals.

ii. $W$ is a non-empty set of possible worlds.

iii. $I$ is the interpretation function.

iv. $\Sigma$ is the refinement function.

v. $\Gamma$ is a set of goals.

\subsubsection{Interpretation function}

$I$ is the interpretation function of the model $M$ assigning an intension $I_{\alpha}$ to each predicate and $I_{\beta}$ to each individual constant as follows:

Definition 3 (Interpretation function $I_{\alpha}$ ) If $\alpha$ is a predicate, then $I_{\alpha}$ is a function such that for each $w \in W: I_{\alpha}(w) \subseteq D$.

Definition 4 (Interpretation function $I_{\beta}$ ) If $\beta$ is an individual constant, then $I_{\beta}$ is a function such that for each $w \in W: I_{\beta}(w) \in D$. 


\subsubsection{Truth}

I write $M, w \models \phi$ for a sentence $\phi$ is true in model $M$ with respect to the world of evaluation $w$.

I also write $\llbracket \phi \rrbracket_{w}$ for the denotation of a sentence $\phi$ at world $w$.

Definition 5 (Sentential truth) $M, w \models \alpha(\beta)$ iff $\forall w \in W: I_{\beta}(w) \in I_{\alpha}(w)$.

Definitions for negation, disjunction, conjunction and implication are classical.

\subsubsection{Refinement function}

The refinement function of the model $M$ is supposed to categorise individuals in the extension of $I_{\alpha}$ at a world $w$ according to different ways in which $I_{\alpha}$ may apply to them. For any $I_{\alpha}$, there will be $n$ number of ways in which it may be applied to an object denoted by $\beta$. Recall that in the current theory the notion of ways has a primarily linguistic motivation in that it is supposed to capture the fact that an objective predicate can be used in different senses.

Definition 6 (Refinement of $I_{\alpha}(w)$ ) Let $\Sigma_{\alpha}$ be a function from $W$ to $n$-tuples of sets of individuals, such that for any world $w$,

- $\Sigma_{\alpha}(w)=\left\langle\sigma_{\alpha}^{1}(w), \ldots, \sigma_{\alpha}^{n}(w)\right\rangle$, where $\sigma_{\alpha}^{i}(w) \subseteq I_{\alpha}(w)$ and $i \in\{1 \ldots n\}$

- $\Sigma_{\alpha}(w)$ is a cover of $I_{\alpha}(w)$ such that the union of the sets in $\Sigma_{\alpha}(w)$ is equal to $I_{\alpha}(w)$, i.e. $\bigcup \sigma_{\alpha}^{i}(w)=I_{\alpha}(w){ }^{24}$

Example 1 Let us assume that $W:\left\{w_{1}, w_{2}, w_{3}, w_{4}, w_{5}\right\}$, and $D:\{a, b, c, d\}$. For each world $w$, let us further assume that the interpretation function $I_{\alpha}$ assigns the following values to the predicate $\alpha$ :

$I_{\alpha}\left(w_{1}\right)=\{a, b, c\}, I_{\alpha}\left(w_{2}\right)=\{a\}, I_{\alpha}\left(w_{3}\right)=\{a, b\}, I_{\alpha}\left(w_{4}\right)=\{a, b, c\}, I_{\alpha}\left(w_{5}\right)=\emptyset$

The application of $\Sigma_{\alpha}$ to $W$, where $n=2$ returns the following values:

$$
\begin{aligned}
& \Sigma_{\alpha}\left(w_{1}\right)=\left\langle\sigma_{\alpha}^{1}\left(w_{1}\right), \sigma_{\alpha}^{2}\left(w_{1}\right)\right\rangle=\langle\{a, c\},\{a, b, c\}\rangle \\
& \Sigma_{\alpha}\left(w_{2}\right)=\left\langle\sigma_{\alpha}^{1}\left(w_{2}\right), \sigma_{\alpha}^{2}\left(w_{2}\right)\right\rangle=\langle\{a\},\{\emptyset\}\rangle \\
& \Sigma_{\alpha}\left(w_{3}\right)=\left\langle\sigma_{\alpha}^{1}\left(w_{3}\right), \sigma_{\alpha}^{2}\left(w_{3}\right)\right\rangle=\langle\{a, b\},\{b\}\rangle \\
& \Sigma_{\alpha}\left(w_{4}\right)=\left\langle\sigma_{\alpha}^{1}\left(w_{4}\right), \sigma_{\alpha}^{2}\left(w_{4}\right)\right\rangle=\langle\{b\},\{a, c\}\rangle \\
& \Sigma_{\alpha}\left(w_{5}\right)=\left\langle\sigma_{\alpha}^{1}\left(w_{5}\right), \sigma_{\alpha}^{2}\left(w_{5}\right)\right\rangle=\langle\emptyset, \emptyset\rangle
\end{aligned}
$$

\subsubsection{Equivalence relations}

Definition 7 (Equivalence relations wrt the meaning of $\alpha(\beta)$ )

$$
w R_{\alpha(\beta)} w^{\prime} \text { iff } I_{\beta}(w) \in I_{\alpha}(w) \leftrightarrow I_{\beta}\left(w^{\prime}\right) \in I_{\alpha}\left(w^{\prime}\right)
$$

\footnotetext{
24 For the definition of set cover see Kelley (2017: 49).
} 
Let $P$ be a partition of $W$, and let $c \in \mathcal{P}$ be a cell of the partition $P$ induced by the meaning of $\alpha(\beta)$. If $w R_{\alpha(\beta)} w^{\prime}$, then both $w$ and $w^{\prime}$ are in the same cell $c$ of the partition $P$ or neither is. Notice that the partition of $W$ induced by the meaning of $\alpha(\beta)$ is equivalent to a disjoint, non-empty set of sets of worlds where $\alpha(\beta)$ is true and where $\alpha(\beta)$ is false, i.e. $P=\{|\alpha(\beta)|,|\overline{\alpha(\beta)}|\}$.

Definition 8 (Equivalence relations wrt $\alpha(\beta)$ and $\Sigma_{\alpha}$ )

$$
w R_{\Sigma_{\alpha}}^{\alpha(\beta)} w^{\prime} \text { iff } \forall i: 1 \leq i \leq n: I_{\beta}(w) \in \sigma_{\alpha}^{i}(w) \leftrightarrow I_{\beta}\left(w^{\prime}\right) \in \sigma_{\alpha}^{i}\left(w^{\prime}\right)
$$

$R_{\Sigma_{\alpha}}^{\alpha(\beta)}$ is an equivalence relation between worlds that holds with respect to the meaning of $\alpha(\beta)$ and a set of ways in which individuals can fall under the property denoted by $\alpha$ (where this set of ways is given by $\Sigma_{\alpha}$ ). It states that two worlds, $w$ and $w^{\prime}$, are equivalent if and only if, for each of the relevant ways, when the individual denoted by $\beta$ falls under that way at $w$, that individual also falls under that way at $w^{\prime}$, and vice versa. So, in our green leaf example, where we just have the two ways in play, this means two worlds would be equivalent when, say, the leaves are painted green at both worlds and naturally green at neither. In the interest of visual clarity in what follows we will write $R$ as a shorthand for $R_{\alpha(\beta)}$ and $\mathcal{R}$ as a shorthand for $R_{\Sigma_{\alpha}}^{\alpha(\beta)}$.

Example 2 Suppose that $\beta=$ the leaf, and $\alpha=$ green. $I_{\beta}(w)=a$ for all worlds in $W$. In that case the interpretation function returns the following values for The leaf is green:

【The leaf is green $\rrbracket_{w_{1}}=1$

«The leaf is green $\rrbracket_{w_{2}}=1$

$\llbracket$ The leaf is green $\rrbracket_{w_{3}}=1$

$\llbracket$ The leaf is green $\rrbracket_{w_{4}}=1$

$\llbracket$ The leaf is green $\rrbracket_{w_{5}}=0$

Let $[w]_{R}$ be an equivalence class generated by $R$. The partition of $W$ then consists of two cells (where $w_{1}$ and $w_{5}$ are class representatives):

$$
\begin{aligned}
{\left[w_{1}\right]_{R} } & =\left\{w_{1}, w_{2}, w_{3}, w_{4}\right\} \\
{\left[w_{5}\right]_{R} } & =\left\{w_{5}\right\}
\end{aligned}
$$

Furthermore, suppose that $\Sigma_{\text {green }}(w)=\left\langle\sigma_{\text {green }}^{1}(w), \sigma_{\text {green }}^{2}(w)\right\rangle$. Then there will be a further fragmentation of the initial partition wrt to these two ways in which $I_{\text {green }}$ may be applied to the object $a$ denoted by the leaf. Assuming $\Sigma_{\text {green }}(w)$ returns the same values as in Example 1, then we have a refined partition of $W$ wrt to the equivalence relation $\mathcal{R}$ as illustrated in Table 1.

\subsubsection{Goal-conduciveness}

In the current framework, given a particular goal $\gamma \in \Gamma$, an agent will evaluate certain partition cells induced by $\mathcal{R}$ as conducive or not to that goal. 
Table 1 Partition of W wrt $\mathcal{R}$

\begin{tabular}{cccc}
\hline$c_{1}: \sigma_{\text {green }}^{1} \wedge \neg \sigma_{\text {green }}^{2}$ & $c_{2}: \neg \sigma_{\text {green }}^{1} \wedge \sigma_{\text {green }}^{2}$ & $c_{3}: \sigma_{\text {green }}^{1} \wedge \sigma_{\text {green }}^{2}$ & $c_{4}: \neg \sigma_{\text {green }}^{1} \wedge \neg \sigma_{\text {green }}^{2}$ \\
\hline$w_{2}$ & $w_{4}$ & $w_{1}$ & $w_{5}$ \\
$w_{3}$ & & & \\
\hline
\end{tabular}

Definition 9 (Goals) Let a goal $\gamma$ be a function from a partition $P$ of $W$ induced by $\mathcal{R}$ to assignments of $c$-value $\{1,0\}$ to each cell $c$ in the partition: $\forall c \in P, \gamma(c) \rightarrow\{1,0\}$.

As discussed above, a successful fulfilment of a goal may depend on a particular way of being F. And, sometimes, it may also be prevented in light of a given way. ${ }^{25}$ In this way c-valuations supervene on goal-relevant properties of ways of being $\mathrm{F}$ holding of an object at a given world. We can formally express the condition of admissibility of c-values as follows:

\section{C-valuations condition}

$\gamma(c)=1$ iff for all $w \in c$ there is some $\sigma_{\alpha}^{i}$ such that $I_{\beta}(w) \in \sigma_{\alpha}^{i}(w)$ and $\sigma_{\alpha}^{i}$ is conducive to $\gamma$.

This condition (henceforth, the $c$-condition) states that a goal $\gamma$ will evaluate a cell $c$ as being conducive to the goal just in case at all the worlds in this cell there is a way $\sigma_{\alpha}^{i}$ holding of an object denoted by $\beta$ which is conducive to $\gamma \cdot{ }^{26}$

Example 3 Suppose that a partition of $W$ induced by $\mathcal{R}$ contains four cells as specified in Table 1. Each cell corresponds to an equivalence class $[w]$ such that:

$$
\begin{aligned}
& c_{1}:\left[w_{2}\right]_{\mathcal{R}}=\left\{w_{2}, w_{3}\right\} \\
& c_{2}:\left[w_{4}\right]_{\mathcal{R}}=\left\{w_{4}\right\} \\
& c_{3}:\left[w_{1}\right]_{\mathcal{R}}=\left\{w_{1}\right\} \\
& c_{4}:\left[w_{5}\right]_{\mathcal{R}}=\left\{w_{5}\right\}
\end{aligned}
$$

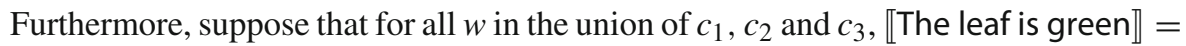

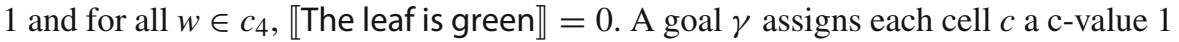
or 0 in accordance with the c-condition. Let us assume $\gamma$ returns the following values:

$$
\begin{aligned}
& \gamma\left(c_{1}\right)=0 \\
& \gamma\left(c_{2}\right)=1 \\
& \gamma\left(c_{3}\right)=1 \\
& \gamma\left(c_{4}\right)=0
\end{aligned}
$$

25 There may also be ways for things to be which are not in themselves conducive to the goal but combined with goal-conducive ways are not damaging to the goal.

26 It may be that for certain cases this condition will need to be stronger so as to, in addition, exclude the ways of being $\mathrm{F}$ that might block the achievement of the goal:

Strong c-condition. $\gamma(c)=1$ iff for all $w \in c$ there is some $\sigma_{\alpha}^{i}$ such that $I_{\beta}(w) \in \sigma_{\alpha}^{i}(w)$ and $\sigma_{\alpha}^{i}$ is conducive to $\gamma$ and there is no $\sigma_{\alpha}^{j}$ such that $I_{\beta}(w) \in \sigma_{\alpha}^{j}(w)$ and $\sigma_{\alpha}^{j}$ is against $\gamma$. 
This valuation map for goal $\gamma$ will be important for determining the truth value of an utterance of The leaf is green at $w$ given a contextually salient goal $\gamma$.

\subsubsection{Contextual truth}

I write $M, w \models_{\gamma} \phi$ for an utterance of $\phi$ is true in model $M$ with respect to the world of evaluation $w$ and the goal of context $\gamma$.

I also write $\llbracket \phi \rrbracket_{\gamma, w}$ for the denotation of a sentence $\phi$ relative to $\gamma$ at world $w$

Definition 10 (Contextual truth)

$$
\begin{aligned}
& M, w \models_{\gamma} \alpha(\beta) \text { iff } \\
& \quad \text { i. } I_{\beta}(w) \in I_{\alpha}(w) \\
& \text { ii. There is no }\left[w^{\prime}\right]_{\mathcal{R}} \in P \text { such that } \gamma\left(\left[w^{\prime}\right]_{\mathcal{R}}\right)>\gamma\left([w]_{\mathcal{R}}\right) \\
& M, w \models_{\gamma} \neg \phi \text { iff } M, w \not \models_{\gamma} \phi \\
& M, w \models_{\gamma}(\phi \vee \psi) \text { iff } M, w \models_{\gamma} \phi \text { or } M, w \models_{\gamma} \psi \\
& M, w \models_{\gamma}(\phi \wedge \psi) \text { iff } M, w \models_{\gamma} \phi \text { and } M, w \models_{\gamma} \psi
\end{aligned}
$$

Let's briefly comment on these clauses.

Atomic sentences The definition states that an utterance of an atomic sentence $p$ relative to the goal of context $\gamma$ is accepted as true at a world $w$ only if $p$ is true at $w$ and $w$ is in the equivalence class $[w]$ generated by $\mathcal{R}$ whose c-value is at least as great as any other class $\left[w^{\prime}\right]$ (i.e. any cell with c-value that is lesser than the c-value of other cells in this partition is excluded from it). Notice that in case no cell is conducive to $\gamma$ the contextual proposition expressed by $p$ will correspond to the minimal proposition expressed by it. ${ }^{27}$

Contextual negation The contextual proposition expressed by $\neg \phi$ is a negation of the contextual proposition expressed by $\phi$. Notice, however, that $\llbracket \neg \phi \rrbracket_{\gamma}$ contains the cells that consist of worlds where $\phi$ is classically false, but it may also contain some cells consisting of worlds where it is classically true but which are not conducive to $\gamma$.

Disjunction The contextual proposition expressed by a disjunctive sentence is true in model $M$ relative to the goal of context $\gamma$ just in case either $\phi$ is true relative to $\gamma$ or $\psi$ is true relative to $\gamma$ (or both).

Conjunction The contextual proposition expressed by a conjunctive sentence is true in model $M$ relative to the goal of context $\gamma$ just in case both $\phi$ is true relative to $\gamma$ and $\psi$ is true relative to $\gamma$.

\footnotetext{
27 The condition (ii) assures that the utterance could be accepted as true when none of the sentence's truth-makers is more conducive to $\gamma$ than others. This is the case, for instance, with (positive) sentences that (contextually) entail or implicate true negations of $\phi$. For example, in the botanist context at the world where the leaf is painted green and naturally red the utterance of These leaves are naturally red is true (and relevant) even though none of its possible truth-makers is conducive to $\gamma$.
} 


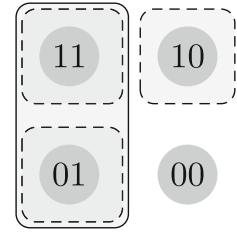

(a) Occasion 1

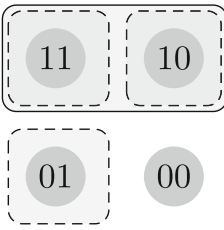

(b) Occasion 2

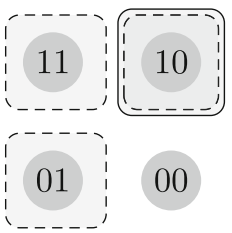

(c) Occasion 3

Fig. 3 Different contextual propositions expressed by The leaves are green

Example 4 Let us assume that the actual world is $w_{2}$ and that the sentence is The leaf is green. As in previous examples $\beta=$ the leaf, $\alpha=$ green, and $\forall w \in$ $W, \llbracket$ the leaf $\rrbracket_{w}=a$. Then, since $w_{2}$ is in the cell $c_{1}$ (see Table 1) and $\gamma\left(c_{1}\right)<\gamma\left(c_{2}\right)$, $M, w \not \nvdash_{\gamma}$ The leaf is green, i.e. the utterance of The leaf is green in model $M$ with respect to the goal of context $\gamma$ is considered false at $w_{2}$.

\section{Applications}

\subsection{Disagreement}

I started this discussion pointing out the intuitive difference between objective and discretionary predicates. I noted that factual statements containing objective predicates, unlike statements of opinion containing discretionary predicates, are normally considered true at a world simpliciter without further relativisation to individual perspectives, etc. However, in an attempt to account for Travis cases I suggested that factual statements are also relative to further factors besides the world of evaluation, namely, to contextually salient goals. So the question is whether the present account preserves the intuitive distinction between objective and discretionary predicates, including the idea of objective disagreement or not.

To answer this question let me first distinguish between some varieties of agreement and disagreement that are relevant to the present discussion. As noted earlier, semantically competent agents are assumed to be in the initial information state, verbally agreeing on what a sentence containing an objective predicate means. Verbal agreement assures that the agents would agree on which worlds constitute the truth set for the sentence. Verbal agreement does not, of course, ensure that the agents will agree in their factual judgements; some agents might be simply ignorant about certain facts which would have an impact on their factual judgements, despite them sharing semantic competence. Still, in Travis cases, the disagreement between agents is not a consequence of any such ignorance: here we assume that the disagreeing parties share relevant knowledge about the world (e.g. they know that the leaves are only painted green). So, if not about meaning or facts, concerning what do agents disagree in these scenarios, and, besides meaning and facts, concerning what exactly there ought to be an agreement between them? Is the variety of disagreement in Travis cases faultless (as in matters of opinion and taste) or is it objective? Does it amount to disagreement at all? 
It is often assumed that for there to be a disagreement between agents in the first place the agents should be contradicting one another, or, minimally, hold opposite (doxastic or non-doxastic) attitudes towards the same (or similar) content. ${ }^{28}$ Here I adopt this simple view of disagreement without, however, wanting to defend any particular theory of predicates of personal taste such as relativism or contextualism. Verbal agreement which assures that agents are not talking past one another is thus necessary for any factual agreement or disagreement. A disagreement is considered faultless if two individuals are contradicting one another, but neither can be said to be at fault (see Kölbel 2004). We have seen earlier that the sense of faultlessness surrounds not only disagreements about taste, but also disagreements about facts once we take into account occasion-sensitivity. Yet, despite this initial appearance of faultlessness, I maintain that occasion-sensitivity properly understood does not destroy the phenomenon of objective, factual disagreement.

To see this, consider again the dialogue between the botanist and the artist concerning naturally red but painted green leaves:

12. (a) The artist (in the artist context): The leaves are green.

(b) The botanist (in the botanist context): The leaves are not green.

I assume that, as competent speakers of English, the artist and the botanist are in verbal agreement about the meanings of green and the leaves. Furthermore, they are both au fait with the relevant facts: for instance, they can both perceive the leaves and they know that they are painted green. The sense of faultlessness here comes from different perspectives that they occupy, which has been cashed out in terms of different goals and purposes. In Fig. 3, I've visually depicted three contextual propositions expressed by The leaves are green relative to three different goals. Let Fig. 3a be the botanist context, and Fig. $3 \mathrm{~b}$ the artist context. ${ }^{29}$ In Fig. 3c I have depicted the contextual proposition determined relative to a goal which requires that the leaves be only painted green. 30

The botanist and the artist agree that the sentence is true at the worlds in cell 11, but they disagree about its truth-value at the worlds in cells 10 and 01 . Notice that, given that the botanist and the artist don't share the same contextual proposition, there is no genuine contextual agreement or disagreement between them. In other words, when communicating, they talk at cross-purposes, and fail to genuinely agree or disagree because they don't share the same contextual goal. Call this variety of agreement and disagreement contextual pseudo-agreement and contextual pseudodisagreement. The fact that there is an appearance of agreement between agents at a world about the truth-value of an utterance does not entail that the agents entertain the same contextual proposition: i.e. although the botanist and the artist pseudo-agree that the utterance of (12) is true in cell 11, they nevertheless express different con-

\footnotetext{
${ }^{28}$ However, see Huvenes (2012) for an argument to the effect that asserting and denying the same proposition is not a necessary condition of disagreement; according to this view, disagreement also arises at the level of implicature. See also: Von Fintel and Gillies (2008), Sundell (2011), De Sa (2015) and Geurts (1998).

${ }^{29}$ Note that this is only for the illustration purposes; I don't claim this depiction necessarily reflects actual goals of an artist or a botanist.

${ }^{30}$ I leave it open as to which goal this may be.
} 
Table 2 Varieties of disagreement: objective predicates

\begin{tabular}{llll}
\hline Disagreement & Shared meaning & Shared proposition & Faultless \\
\hline Verbal & No & - & - \\
Pseudo & Yes & No & - \\
Genuine & Yes & Yes & No \\
\hline
\end{tabular}

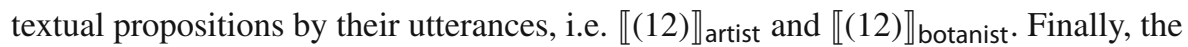
botanist's proposition and the proposition in Fig. 3c never intersect: there will be no contextual pseudo-agreement between the agents entertaining these propositions, although they still verbally agree on lexical meanings of green and the leaves.

So far I explained what verbal and contextual pseudo-agreement and -disagreement consist in. When, according to the present account, would two agents genuinely agree or disagree in a given context? Let's compare two versions of the dialogue between the botanist and the artist, in (12) and (13), where (13) resembles the original Travis case.

13. (a) The artist (in the botanist context): The leaves are green.

(b) The botanist (in the botanist context): No, the leaves are not green.

In the dialogue (12), the artist and the botanist are not part of the same context: they don't share the same goal. Intuitively they both seem to be saying something true relative to their goals, hence the sense of faultlessness. The contrast between the dialogues in (12) and (13) can be accounted for by the difference in the activation of relevant c-maps. In (12) either (i) the artist's information state does not include the c-map for the botanist's goal; (ii) she's not sufficiently aware of the botanist's goal and so is unable to activate the relevant c-map; or (iii) she might be mistaken about what is conducive to the botanist's goal (e.g. her c-map isn't accurate). In any case, she is unable to share the botanist's proposition and so they communicate at cross-purposes. However, assuming the artist knows enough about the botanist's goal and what sort of a green leaf is required for it (viz. she activates a relevant and accurate c-map for the botanist's goal), she ought to accept (if rational) that an utterance (13a) is false at the worlds in cell 10. Given that the artist in the dialogue (13) could and should evaluate the cell (for goal-conduciveness) from the point of view of the botanist's goal, she is at fault as regards the truth-valuation of the utterance. Thus, here we have a case of objective disagreement because, with respect to the worlds in cell 10, one party (the artist) asserts that the leaves are green in a way that is suitable for the botanist's purpose, thus expressing 【(13)】 botanist, and the other party (the botanist) claims that the leaves are not green in a way that is suitable for her purpose, expressing $\llbracket \neg(13) \rrbracket_{\text {botanist. In }}$ Table 2 I have summarised three varieties of disagreement for sentences containing objective predicates and how they relate to shared semantic and pragmatic knowledge.

Let us now see how this classification connects with sentences containing discretionary predicates. My first observation is that, unlike factual judgements, judgements of taste possibly depend on subjective, phenomenal experiences and non-doxastic attitudes $^{31}$ whose contents are impervious to public scrutiny. A fortiori, the requirement

31 See Huvenes (2014). 
to retract one's judgement in the face of acquiring new, contextually relevant (factual) knowledge does not apply to matters of taste in the same way as it applies to matters of fact. To appreciate this point consider the following two dialogues in which A learns some new relevant facts in light of which A may retract the original judgement.

\section{$B$ needs a green leaf to conduct an experiment; the leaf is only painted green.}

14. (a) A: The leaf is green.

(b) B: No, the leaf is not green.

(c) B tells A that a painted green leaf in not conducive to conducting a botanical experiment.

(d) A: *I see, but the leaf is green!

$A$ and $B$ both dislike pork, they don't find it tasty.

15. (a) A: This chilli is tasty.

(b) B: No, this chilli is not tasty.

(c) B tells A that the chilli contains pork.

(d) A: I see, but the chilli is tasty!

Whilst the response in (14d) seems inappropriate, the response in (15d) is intuitively acceptable. I suggest that this is due to the fact that learning a new fact about the chilli need not affect A's attitude toward the chilli and his personal gustatory experience on which her judgement of taste is grounded. By contrast, learning a new piece of information about the relation between greenness and botanical experiments ought to make A retract her original judgement. When A and B disagree as to whether the chilli is tasty the best they could do is acknowledge that their attitudes towards the world are different. Still no matter what they do (how much factual information about the world or each other they obtain), there is no rational expectation or requirement for A to put himself in B's perspective (and vice versa) and acknowledge that (and in which way) A's standard of taste is wrong (false) and B's is correct. Contrast this to matters of fact where there $i$ s such a requirement once both agents are assumed to be aware of the contextual goal and have activated relevant valuation maps.

A second, related observation: although factual judgements like judgements of taste are, indeed, dependent on further factors over an above the world of evaluation, the nature of these factors crucially differs. In particular, whilst the latter are dependent on subjective standards of taste (or sets thereof), ${ }^{32}$ the former depend on activities and goals that are widely shared and are, in principle, accessible by all rational creatures. Anyone with an appropriate knowledge base (including know-how) about a certain practice could come to appreciate what's required to fulfil its goals successfully. But no knowledge of facts is in itself sufficient to bring about appreciation that something yucky or boring is, as a matter of fact, tasty or fun because there is no fact that decides these matters. So, even though, prima facie, occasion-sensitivity seems to temporarily blur the intuitive contrast between objective and discretionary predicates, the contrast is still there insofar as objective perspectives (goals and valuation maps) are accessible to all rational agents and subjective perspectives (personal tastes) aren't.

\footnotetext{
${ }^{32}$ Huvenes (2012) points out that on more sophisticated versions of contextualism it's standards of a wider community that are relevant for judgements of taste (Huvenes 2012: 172). Nonetheless increasing the number of individuals as the value of the 'judge' parameter doesn't seem to make judgements of taste less subjective.
} 


\subsection{Cancellability}

Cancellability is a feature of utterances usually associated with conversational implicatures where the intended content to be communicated by an utterance is something that is implied by uttering a sentence in a given context rather than something that the sentence would semantically express (meaning what it does). By saying that I am French I (may) imply in a context that I am a good cook (and a number of other things). ${ }^{33}$ In the following example, (16b) conversationally implicates (16c), which here spells out the content that the speaker intends to communicate in the context.

16. (a) Can you cook?

(b) I am French.

(c) $\rightsquigarrow$ I am a good cook

Any implicated content such as the one in (16b) may be explicitly cancelled if the speaker wants to make sure that the hearer doesn't interpret (16b) as (16c).

17. I am French but I am not a good cook.

Whilst expected implicatures may be felicitously cancelled in this way, we cannot cancel what the sentence semantically expresses without a contradiction as illustrated in (18).

\section{8. *I am French but I am not French.}

Now even though I maintain it would be wrong to assimilate the phenomenon of occasion-sensitivity to that of conversational implicature, it is still important to note that in Travis cases the contextually expected interpretation of an utterance $i s$ cancellable without a contradiction. In (19) I assume by now the familiar set-up where the salient leaves are painted green and naturally red and the salient goal is to perform a botanical experiment.

19. (a) Botanist: Are the leaves green?

(b) Pia: The leaves are green, but they are not naturally green.

Unlike in the original Travis case where Pia's response (viz. that the leaves are green) is intuitively unacceptable (and I offered an explanation of why this is so), once the contextually expected answer is made explicit then Pia's response in (19b) seems more acceptable. How does the current theory account for this intuition?

First, the cancellation in (19b) indicates a certain sort of disagreement. The response can be read in several different ways: (i) as a rejection of the expected definition of green (as a case of meta-linguistic or verbal disagreement), (ii) as a rejection of the contextually expected interpretation of green which presupposes the botanist's goal (meta-contextual disagreement), and (iii) as a factual claim. I assume that, in this particular example, Pia has activated the adequate valuation map and is aware of the botanist's goal given that she indicates the contextually expected reading of the expression green leaves by using a more specific form, namely, naturally green

\footnotetext{
33 See Recanati (2004).
} 
leaves. However, instead of simply denying that the leaves are green (i.e. saying that the leaves are not green), Pia adds more material in the second conjunct (explicating the expected interpretation) and also asserts (in the first conjunct) that they are green in some other way where this is not the way expected in this context. So in (19b) the expected interpretation of These leaves are green is cancelled but it is also asserted that the leaves count as being green in some other way, for some other unknown purpose. The remark thus appears to be both factual and meta-contextual ${ }^{34}$ in as much attention is drawn to another possible use of green which is not the one that the botanist would expect on this occasion.

Although (19b) is acceptable it is not entirely appropriate. I maintain that its acceptability stems from the fact that (i) the contextually expected reading has been acknowledged, if not endorsed, (ii) other uses of green are available (although not suitable for this purpose). Nonetheless, (19b) is inappropriate since it rejects the contextually expected interpretation. This kind of cancellation is thus justified only when there is a reasonable assumption that the interlocutor may be interested in other possible uses of green, or where there are two or more conflicting goals on an occasion. Otherwise the cancellation comes across as inappropriate as it's imposing the interpretation that is not relevant to her interlocutor.

\section{Conclusion}

In this paper I proposed an occasion-sensitive semantics for declarative sentences containing objective predicates as a way to account for the phenomenon observed in Travis cases. Travis cases indicate that in many cases speakers would accept only a subset of the set of worlds where a sentence is true as its truth-maker on a given occasion. The suggested explanation for occasion-sensitivity exploits the idea that the set of worlds in which a sentence is true has a more complex structure as a result of different ways in which the sentence is made true. I proposed to capture this formally by means of a partition semantics. Furthermore, I suggested that the main pragmatic factor guiding interpretation is a contextually salient domain goal. Given that rational agents may pursue different goals on different occasions, I argued that their information states can be seen as being equipped with particular c-maps, which in turn play a role in truth-evaluations of utterances on particular occasions. Additionally, I have shown how the proposed account applies to certain cases of disagreement and cancellability, and I argued that the current approach preserves the intuitive distinction between discretionary and objective predicates.

Based on the account I defended in this paper there is one particular avenue for future work that I am keen to explore. The empirical focus of many accounts of occasionsensitivity has thus far been restricted to declarative sentences. I believe, however, that the phenomenon is more general and that it affects the interpretation of other sentential forms besides declaratives. My ambition is to try to extend the proposed formal model to interrogative and imperative sentences. For now, however, I hope to have shown how the interpretation of declarative sentences containing objective

\footnotetext{
34 See Sundell (2011) and Zeman (2017) for a more detailed discussion of this variety of disagreement.
} 
predicates crucially depends on wider goal-directed projects: insofar as such projects change from one occasion to the next so do our expectations as to what our sentences say.

Open Access This article is distributed under the terms of the Creative Commons Attribution 4.0 International License (http://creativecommons.org/licenses/by/4.0/), which permits unrestricted use, distribution, and reproduction in any medium, provided you give appropriate credit to the original author(s) and the source, provide a link to the Creative Commons license, and indicate if changes were made.

\section{References}

Austin, J. L. (1975). How to do things with words. Oxford: Oxford University Press.

Borg, E. (2004). Minimal semantics. Cambridge: Cambridge University Press.

Borg, E. (2012). Pursuing meaning. Oxford: Oxford University Press.

Cappelen, H., \& Lepore, E. (2005). Insensitive semantics: A defense of semantic minimalism and speech act pluralism. Oxford: Blackwell.

Carston, R. (2002). Thoughts and utterances: The pragmatics of explicit communication. Oxford: Wiley.

Chomsky, N. (2000). New horizons in the study of language and mind. Cambridge: Cambridge University Press.

Ciardelli, I., Groenendijk, J., \& Roelofsen, F. (2013). Inquisitive semantics: A new notion of meaning. Language and Linguistics Compass, 7(9), 459-476.

Ciardelli, I., Groenendijk, J., \& Roelofsen, F. (2015). On the semantics and logic of declaratives and interrogatives. Synthese, 192(6), 1689-1728.

Ciardelli, I. A., \& Roelofsen, F. (2015). Inquisitive dynamic epistemic logic. Synthese, 192(6), 1643-1687.

Coppock, E. (2018). Outlook-based semantics. Linguistics and Philosophy, 41(2), 125-164.

Davies, A. (2017). Elaboration and intuitions about disagreement. Philosophical Studies, 174(4), 861-875.

De Sa, D. L. (2015). Expressing disagreement: A presuppositional indexical contextualist relativist account. Erkenntnis, 80(1), 153-165.

Geurts, B. (1998). The mechanisms of denial. Language, 74(2), 274-307.

Giberman, D. (2016). Moving parts: A new indexical treatment of context-shifting predication. Synthese, 193(1), 95-124.

Groenendijk, J., \& Roelofson, F. (2009). Inquisitive semantics and pragmatics. Paper presented at the Workshop on Language, Communication, and Rational Agency, Stanford, May 30-31, 2009. http:// www.illc.uva.nl/inquisitive-semantics.

Groenendijk, J. \& Stokhof, M. (1984). Studies on the semantics of questions and the pragmatics of answers. $\mathrm{Ph} . \mathrm{D}$. thesis, University of Amsterdam.

Hawke, P. (2017). Theories of aboutness. Australasian Journal of Philosophy, 96, 1-27.

Huvenes, T. T. (2012). Varieties of disagreement and predicates of taste. Australasian Journal of Philosophy, 90(1), 167-181.

Huvenes, T. T. (2014). Disagreement without error. Erkenntnis, 79(1), 143-154.

Kaplan, D. (1989). Demonstratives. In J. Almog, J. Perry, \& H. Wettstein (Eds.), Themes from Kaplan (pp. 483-540). Oxford: Oxford University Press.

Kelley, J. L. (2017). General topology. New York: Courier Dover Publications.

Kennedy, C., \& McNally, L. (2010). Color, context, and compositionality. Synthese, 174(1), 79-98.

Kölbel, M. (2004). Faultless disagreement. In Proceedings of the Aristotelian society (Vol. 104, pp. 53-73). Oxford: The Oxford University Press.

Lasersohn, P. (2005). Context dependence, disagreement, and predicates of personal taste. Linguistics and philosophy, 28(6), 643-686.

MacFarlane, J. (2014). Assessment sensitivity: Relative truth and its applications. Oxford: OUP.

Moravcsik, J. (1994). Is snow white? In P. Humprey (Ed.), Patrick Suppes: Scientific philosopher (Vol. 3, pp. 71-87). Dordrecht: Kluwer.

Pagin, P. (2005). Compositionality and context. In G. Preyer \& G. Peter (Eds.), Contextualism in philosophy: Knowledge, meaning, and truth (pp. 303-348). Oxford: Oxford University Press. 
Pagin, P. \& Pelletier, J. (2007). Content, context and composition. In G. Preyer \& G. Peter (Eds.) Contextsensitivity and semantic minimalism: Essays on semantics and pragmatics (pp. 25-62). Oxford: Oxford University Press.

Pietroski, P. (2005). Meaning before truth. In G. Preyer \& G. Peter (Eds.), Contextualism in philosophy: Knowledge, meaning, and truth (pp. 253-299). Oxford: Oxford University Press.

Recanati, F. (2004). Literal meaning. Cambridge: Cambridge University Press.

Recanati, F. (2010). Truth-conditional pragmatics. Oxford: Clarendon Press.

Roberts, C. (1996). Information structure in discourse: Towards an integrated formal theory of pragmatics. In J.-H. Yoon \& A. Kathol (Eds.), Ohio State University Working Papers in Linguistics (Vol. 49, pp. 91-136). Columbus, OH: Ohio State University Publications.

Roberts, C. (2012). Information structure: Afterword. Semantics and Pragmatics, 5(7), 1-19.

Rothschild, D., \& Segal, G. (2009). Indexical predicates. Mind \& Language, 24(4), 467-493.

Schoubye, A. J., \& Stokke, A. (2015). What is said? Noûs, 50(4), 759-793.

Searle, J.R. (1980). The background of meaning. In: J.R. Searle, F. Kiefer \& M. Bierwisch (Eds.), Speech act theory and pragmatics. Texts and studies in linguistics and philosophy (Vol. 10, pp. 221-232). Dordrecht: Springer.

Stojanovic, I. (2007). Talking about taste: Disagreement, implicit arguments, and relative truth. Linguistics and Philosophy, 30(6), 691-706.

Sundell, T. (2011). Disagreements about taste. Philosophical Studies, 155(2), 267-288.

Travis, C. (1978). Meaning versus truth. Dialogue, 17(03), 401-430.

Travis, C. (2000). Unshadowed thought: Representation in thought and language. Cambridge, MA: Harvard University Press.

Travis, C. (2008). Occasion-sensitivity: Selected essays. Oxford: Oxford University Press.

Travis, C. (2009). Thought's footing: A theme in Wittgenstein's philosophical investigations. Oxford: Oxford University Press.

Van Rooy, R. (2003). Questioning to resolve decision problems. Linguistics and Philosophy, 26(6), 727763.

Vicente, A. (2012). On travis cases. Linguistics and Philosophy, 35(1), 3-19.

Von Fintel, K., \& Gillies, A. S. (2008). CIA leaks. Philosophical Review, 117(1), 77-98.

Yablo, S. (2014). Aboutness. Princeton: Princeton University Press.

Yablo, S. (2017). Precis of aboutness. Philosophical Studies, 174(3), 771-777.

Zeman, D. (2017). Contextualist answers to the challenge from disagreement. Phenomenology and Mind, $12,62-73$.

Publisher's Note Springer Nature remains neutral with regard to jurisdictional claims in published maps and institutional affiliations. 